ENFOQUES JURIDICOS

REVISTA MULTIDISCIPLINAR DEL CEDEGS

ISSN 26832070

Número 04

julio-diciembre 2021
Artículo: "Enseñanza del Derecho en la Pandemia: La clase magistral interactiva en la virtualidad"

Cristina Cázares Sánchez

DOI: https://doi.org/10.25009/ej.v0i04.2569

\title{
Enseñanza del Derecho en la Pandemia: La clase Magistral interactiva en la virtualidad
}

Recibido 08 febrero 2021-Aceptado 26 marzo 2021.

\author{
Cristina Cázares Sánchez* \\ Universidad Nacional Autónoma de México-CDMX \\ ccazaress@derecho.unam.mx
}

RESUMEN: La pertinencia de la clase magistral ha sido continuamente cuestionada como estrategia para la Enseñanza del Derecho, en concreto dentro del sistema presencial. Con la emergencia sanitaria a nivel Mundial, este sistema se ha mudado al sistema en línea, predominando las clases sincrónicas como la forma habitual de enseñanza en las Aulas Virtuales. Sin embargo, la estrategia que siguen es la misma de la clase magistral tradicional, por ello, se retoman en este artículo los argumentos, metodología propuestos por varios autores en la Enseñanza Superior, para transformar a la clase magistral en interactiva, donde se rompe el esquema del monólogo, fomentar la participación del alumnado mediante determinadas herramientas tecnológicas.

Se propone una estructura de la clase magistral adecuada a la enseñanza en línea emergente, no se trata de una propuesta
ABSTRACT: The relevance of the master class has been continuously questioned as a strategy for the Teaching of Law, specifically within the face-to-face system. With the health emergency worldwide, this system has moved to the online system, with synchronous classes prevailing as the usual way of teaching in Virtual Classrooms. However, the strategy that they follow is the same as the traditional master class, therefore, in this article the arguments, methodology proposed by various authors in Higher Education, are taken up to transform the master class into interactive, where the monologue scheme disappears and the interactive scheme encourages student participation through certain technological tools. A structure of the master class suitable for emerging online teaching is proposed, it is not a proposal for Distance Learning, which has its own teaching-learning methodology.

* Profesora Tiempo Completo Facultad de Derecho, UNAM.

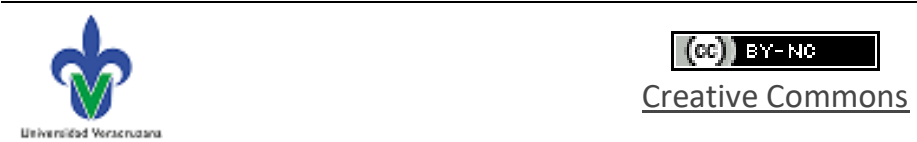


ENFOQUES JURÍDICOS

REVISTA MULTIDISCIPLINAR DEL CEDEGS

ISSN 26832070

Número 04

julio-diciembre 2021
Artículo: “Enseñanza del Derecho en la Pandemia: La clase magistral interactiva en la virtualidad"

Cristina Cázares Sánchez

DOI: https://doi.org/10.25009/ej.v0i04.2569

para la Enseñanza a Distancia, que tiene su

Keywords: $\quad$ Teaching, Pandemic, propia metodología de enseñanzaaprendizaje. Masterclass, Interactivity, ICTS, Virtual

Palabras Clave: Enseñanza; Pandemia, Clase magistral; Interactividad; TICS; Aulas Virtuales. Classrooms.

SUMARIO: Introducción; 1. La clase magistral interactiva; 2. El discurso del docente; 3. La estructura de una clase magistral interactiva; Conclusiones; Fuentes de consulta.

\section{Introducción}

Durante muchos años se ha criticado a la clase magistral o tradicional en la enseñanza del Derecho (Witker, 1985) y se debe a una mala fama que se da a partir de una interpretación meramente unilateral de la clase, es decir, la clase magistral es asimilada a un monólogo de duración similar al tiempo institucional de la clase, de allí, que (Amilcar, Laguyás et Palacios, 2011) en la encuesta que realizaron en la Facultad de Derecho de la Universidad Nacional del Mar del Plata, encontraron los siguientes comentarios:

* La sobreabundancia de clases magistrales a lo largo de toda la carrera. * El método expositivo sin abrir el juego a disentir con los postulados de la cátedra, que genera un clima de aburrimiento, desinterés y hasta hostilidad. * Evaluaciones improvisadas, consignas poco claras y que son redactadas minutos antes del comienzo de la prueba.

* La exaltación de la memorización, y por ende el ahogamiento de todo intento de pensamiento crítico. ${ }^{*}$ Las clases donde se leen los códigos o se repiten textuales pasajes de los libros propuestos por las cátedras. * ...la clase netamente teórica... Se pierde atención, la motivación... Mucho más en aquellas en donde el docente se limita a dictar. * El dictado, que no incentiva el pensamiento y no logra alcanzar el conocimiento en su plenitud. (Amilcar, Laguyás et Palacios, 2011: 324)

Por tanto, se asimila a la clase magistral con el dictado, la memorización, lectura de códigos o libros, donde no hay diálogo ni participación activa del alumnado, sin embargo, existe evidencia teórica y práctica para rescatar y reformular la clase magistral, que además se sigue utilizando aún de forma virtual.

En la Escuelas de Derecho la clase magistral expositiva es la forma más recurrente de llevar a cabo las clases, aún en la nueva modalidad híbrida o presencial-virtual o sincrónica, no obstante, aún este modelo unívoco tiene:

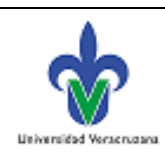

pp. 27-38

LEVEe 
ENFOQUES JURÍDICOS

REMSTA MULTIDISCIPLINAR DEL CEDECS

ISSN 26832070

Número 04

julio-diciembre 2021
Artículo: "Enseñanza del Derecho en la Pandemia: La clase magistral interactiva en la virtualidad"

Cristina Cázares Sánchez

DOI: https://doi.org/10.25009/ej.v0i04.2569

...una valoración positiva indica que estos abogados, formados en distintas escuelas de Derecho, consideran como una contribución a su proceso de formación el que determinados académicos fueran oradores interesantes que les dieron a conocer volúmenes importantes de materia... (Elgueta y Palma, 2014: 913)

Así las clases magistrales son funcionales (Charaja, 2014) en el entorno masivo de las Universidades, en especial en las Facultades de Derecho, donde existe abundante demanda. Sin embargo, para estar acordes con un enfoque cognitivo constructivista (Rénes, 2018) no puede continuar con las características unilaterales y se debe convertir en una clase magistral interactiva (Morell, 2014).

Metodología:

Cabe realizarse la pregunta de investigación ¿Cómo adecuar las clases magistrales interactivas dentro de la virtualidad sincrónica derivada de la Pandemia? ¿Qué estrategias y herramientas tecnológicas pueden utilizarse para ello?

Una de las estrategias más recomendadas para adoptar un enfoque centrado en el estudiante es la gamificación (Navas, Rodríguez y Gómez, 2019). Si bien, existe la posibilidad de adoptar diversas estrategias para dinamizar la clase, en los programas de estudio de la licenciatura en Derecho existen temas cuya trascendencia o especialización los convierten en conceptos fundamentales.

Estos conceptos, deben ser abordados por una estrategia como la clase magistral dada por el docente o un experto, no obstante, para evitar caer en la falta de participación del alumnado o un monólogo extenso, existen técnicas para incluir preguntas y fomentar la atención de los estudiantes.

El objetivo del presente, es reflexionar acerca de la pertinencia de la clase magistral interactiva para abordar conceptos jurídicos que el docente considere fundamentales en las sesiones virtuales-presenciales o sincrónicas que se llevan a cabo como consecuencia de la adaptación de los sistemas presenciales de Enseñanza del Derecho por la Pandemia.

Lo anterior lo realizó a través de la metodología para una clase magistral interactiva propuesta por San Martín, Heurtebize, Veyrunes y Ría, 2021 que consiste en 1) tiempo de exposición; 2) presentación de la pregunta; 3) trabajo individual y primera votación y 4) trabajo colaborativo y segunda votación y 5) un nuevo tiempo de enseñanza o explicación (P10) y desarrollar una propuesta de interacción en la virtualidad.

En el apartado siguiente procederé a describir la propuesta de clase magistral interactiva adaptada a los ambientes virtuales (AV).

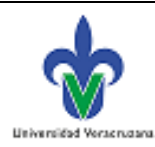

pp. 27-38 
ENFOQUES JURÍDICOS

REVISTA MULTIDISCIPLINAR DEL CEDECS

ISSN 26832070

Número 04

julio-diciembre 2021
Artículo: "Enseñanza del Derecho en la Pandemia: La clase magistral interactiva en la virtualidad"

Cristina Cázares Sánchez

DOI: https://doi.org/10.25009/ej.v0i04.2569

\section{La clase magistral interactiva}

La primera pregunta que surge es ¿Cuándo utilizar una clase magistral? la pertinencia esta en la Enseñanza del Derecho, sin caer en el modelo tradicional pasivo, pues bien, Elgueta y Palma (2014) realizan una distinción muy clara de las clases magistrales aplicadas en las escuelas de Derecho, a saber:

1. La clase magistral propiamente tal o experta, dadas en el contexto de eventos, congresos, etcétera, son propiamente las conferencias magistrales.

2. Clase magistral expositiva dada por el profesor en el salón de clases sin diálogo

3. Clase magistral de profesores memorables, donde el o la docente tienen una habilidad de exposición e interacción con los alumnos para lograr un ambiente de enseñanza-aprendizaje. (Elgueta y Palma, 2014: 909-914)

La interactividad sólo puede darse en el tercer tipo de clase magistral, se trata de volver trascendente en términos de Elgueta y Palma a la clase.

¿Qué características hacen a una clase magistral traspasar el olvido y quedarse en el aprendizaje, en la memoria del estudiante en Derecho?

Sánchez y Barba (2019:91-93) realizan las siguientes recomendaciones desde la Neurociencia para impartir una clase magistral memorable:

1. Entrar en clase con una sonrisa y energía.

2. Procurar que los estudiantes interactúen entre ellos

3. Producir sorpresa en los estudiantes, debe realizarse al principio

4. Contar historias para despertar la curiosidad

5. No desmotivar

6. Mantener la atención del estudiante, para lo cual proponen no exponer en monólogo más de 15 minutos,

7. Realizar estrategias de aprendizaje, como resolución de problemas cortos, actividad física en intervalos cortos.

8. Formular preguntas a los estudiantes para resolver en lo individual y discutir en grupo. Este punto concuerda con la metodología propuesta por San Martín et al, (2021) en realizar preguntas con una fase de respuesta en lo individual y otra de trabajo colaborativo.

9. Usar ejemplos

10. Tener un buen trato hacia los estudiantes

11. Ayudar a la memorización de conceptos.

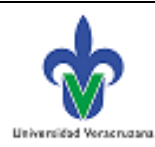

pp. 27-38 
ENFOQUES JURIDICOS

REVISTA MULTIDISCIPLINAR DEL CEDEGS

ISSN 26832070

Número 04

julio-diciembre 2021
Artículo: "Enseñanza del Derecho en la Pandemia: La clase magistral interactiva en la virtualidad"

Cristina Cázares Sánchez

DOI: https://doi.org/10.25009/ej.v0i04.2569

12. Combinar imágenes y textos en las diapositivas (si se usan)

13. Practicar meditación en el aula

Si bien, es difícil llevar a cabo todas las recomendaciones tales como estimular el ejercicio físico y practicar meditación, aunque sí puede administrarse el tiempo que los alumnos pasan sentados ya sea en el aula o en dispositivo móvil, una de las prácticas que realiza algunas universidades tales como la Université de Montréal en los seminarios doctorales, es el trabajo de 40 minutos por 15 de descanso cronometrados, ya que la duración institucional de los mismos es de 3 horas la sesión, lo cual coincide con algunos cursos de posgrado en la Escuelas de Derecho.

¿Cómo aplicar estas recomendaciones en un curso virtual sincrónico?

Según la Guía pedagógica para el desarrollo de clases en entornos virtuales (2020) de la Unidad de Innovación Educativa de la Universidad de Santiago de Chile, las clases virtuales no deben superar los 60 minutos (P.10) lo cual coincide con lo establecido por Sánchez y Barba (2019) respecto a la capacidad de atención en intervalos de 15 minutos de exposición unilateral y administración de actividades para lograr 50 minutos de clase magistral interactiva(P:92)

En la Educación a Distancia, propiamente dicha, me refiero no a la derivada de la mudanza virtual de lo presencial ocasionada por la Pandemia, el tiempo tiene una concepción diferente, y lo sincrónico, ocasionalmente es utilizado como un recurso educativo, ya que las actividades asincrónicas permiten:

...un proceso educativo más longevo en el sentido de que la virtualidad favorece la combinación de las diferentes tareas personales-profesionales, familiares, sociales, etc.-a lo largo del ciclo vital, porque distribuye el tiempo de estudio de un modo más flexible que la enseñanza presencial. En segundo lugar, apreciamos la posibilidad, no siempre aprovechada, que nos ofrecen las TIC de seguir un proceso educativo más intenso e interactivo, en el sentido de que proporciona un mayor tiempo de relación didáctica entre profesor y alumnado. (Badia, 2005: P8)

Así, la clase magistral interactiva es aplicable a las sesiones virtuales sincrónicas del sistema presencial on line, donde el tiempo es físico o real y debe administrarse para lograr los objetivos del proceso de enseñanza-aprendizaje, donde el tiempo se mezcla simultáneamente con el tiempo de la familia, las actividades cotidianas, el ruido del entorno, en fin, con todas las circunstancias ambientales que envuelven al docente y al alumno, incluyendo el cansancio físico y mental que conlleva estar sentado frente a un dispositivo tecnológico y la imposibilidad de desarrollar completa atención en todo momento durante sesiones de más de 3 horas diarias.

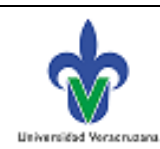

pp. 27-38 
ENFOQUES JURIDICOS

REMSTA MULTIDISCIPLINAR DEL CEDEGS

ISSN 26832070

Número 04

julio-diciembre 2021
Artículo: "Enseñanza del Derecho en la Pandemia: La clase magistral interactiva en la virtualidad"

Cristina Cázares Sánchez

DOI: https://doi.org/10.25009/ej.v0i04.2569

De acuerdo con Hodelín, Reyes, Hurtado y Batista (2016) pasar de 6 a 8 horas frente a una computadora o dispositivo similar, incluyendo no solo el tiempo de clases sino otras actividades, pueden ocasionar o agravar el riesgo de cáncer, diabetes, problemas cardíacos, obesidad, estrés ocular computacional, desórdenes de tendón, problemas de espalda, cuello y hombros, disminución de la vista, debilitamiento muscular y lesiones por esfuerzo repetitivo. Por tanto, se debe valorar la duración de clases sincrónicas durante el día tanto para el docente como para los alumnos y evitar que circunstancias de salud aparezcan en perjuicio de ambos.

Una estrategia recomendada es seleccionar los temas que serán expuestos en una clase magistral interactiva y alternarla con actividades asincrónicas, que permitan la construcción de ese conocimiento más longevo como se da en la educación a distancia.

Planear una clase magistral interactiva

a) Selección del tema:

El docente elegirá el tema conceptual más adecuado para exponer durante intervalos de máximo 15 minutos de monólogo e interrumpir con actividades.

b) Realización de material audiovisual.

Aunque no es obligatorio, ayuda a la comprensión del tema como lo demuestra el estudio realizado por Ramello (2019). Desde esta presentación se pueden vincular las actividades interactivas que se presentan en los apartados de trabajo individual y el trabajo colaborativo.

c) Selección de la herramienta tecnológica para crear la actividad interactiva, es importante señalar que se recomienda el uso de herramientas gratuitas, alta compatibilidad en diversos dispositivos electrónicos y privilegiar las que utilicen menor cantidad de datos de internet considerando las circunstancias sociales y económicas de los docentes y alumnos.

d) Seleccionar la estrategia didáctica, estructurar las actividades y determinar las tareas que llevarán a cabo los alumnos, considerando el tiempo de la sesión.

e) Determinar las evidencias y el cómo va a evaluar las actividades realizadas durante la clase magistral en el total de la calificación del curso.

Como puede observarse, se trata de una labor docente bastante compleja el preparar una clase de este tipo, por ello no debieran ser cotidianas, es decir, conectarse cada día de clase como si se estuviese en el sistema presencial con la finalidad de realizar el dictado de los conceptos o solo exponer el tema sin participación de los alumnos o una participación limitada a una o dos preguntas, al final de la clase.

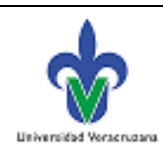

pp. 27-38 
ENFOQUES JURIDICOS

REMSTA MULTIDISCIPLINAR DEL CEDEGS

ISSN 26832070

Número 04

julio-diciembre 2021
Artículo: "Enseñanza del Derecho en la Pandemia: La clase magistral interactiva en la virtualidad"

Cristina Cázares Sánchez

DOI: https://doi.org/10.25009/ej.v0i04.2569

\section{El discurso del docente}

En la clase magistral el discurso del docente es el centro de la motivación, la exposición central, entonces los argumentos utilizados son esenciales:

Los profesores, comunican no sólo realidades, sino que comunican sus propias experiencias de reconstrucción de esas realidades...

La transmisión de esta experiencia, añadida a la comprensión del contenido, refuerza la comprensión que el profesor busca. Creemos interpretar adecuadamente a Habermas (1979), si afirmamos que él encuentra la clave de la transmisión convincente de la experiencia del profesor en la fuerza ilocutiva de sus actos de habla (Tarabay, 2007: 33)

Una clase magistral interactiva debe realizarse en forma de diálogo, sin embargo, fomentar el diálogo implica planeación y estrategia en el discurso del docente, no es un discurso en forma de monólogo sino un discurso autoadministrado con pausas, con elementos que fomenten la respuesta del oyente, es decir, del alumno:

Para lograr los objetivos de la interacción, además de ser aceptado como interlocutor válido, los participantes tienen que conocer (y aprender en situación) las formas del lenguaje, sus funciones y el contexto constitutivo del encuentro (Tronchoni, Izquierdo, Anguera, 2018: 85)

El lenguaje verbal con los argumentos, entonación, ritmo, pausas y silencios, ayudan al docente a construir un ambiente dentro del aula virtual, por ello se requiere que se tengan dos elementos fundamentales, una iluminación adecuada, así como un audio claro, pero también influye el lenguaje no verbal del docente, por ello la realización de gestos expresa las emociones dentro del aula y puede influir positiva o negativamente en el aprendizaje:

La mirada de los docentes es uno de los canales con mayor potencial expresivo para la manifestación de sus propósitos y sentimientos. Es difícil describir con exactitud la gama de miradas observadas, porque la mayoría de las veces se acompañan con otros recursos expresivos como las cejas, la boca y movimientos de la cabeza... (Shabilico, 2012: 115)

Debido a la postura al impartir clases en línea, donde el docente se encuentra sentado frente a la computadora o cualquier otro dispositivo, se expresa dentro del aula virtual de una forma más específica a su rostro y gesticulación que en una aula física, ya que el resto del cuerpo quita la atención del alumnado hacia la cara tal y como se realiza el enfoque en una videocámara. Por ello, es recomendable observarse al espejo para prevenir interpretaciones distintas de un gesto realizado, que haga perder fuerza a los argumentos del discurso del docente o desvíen la atención.

Respecto a los materiales audiovisuales como las presentaciones multimedia son altamente recomendable para reforzar los argumentos del discurso docente, aun cuando no contengan elementos animados, basta que se cuide la proporción entre imagen, texto,

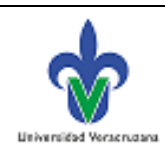

pp. 27-38 
ENFOQUES JURIDICOS

REVISTA MULTIDISCIPLINAR DEL CEDEGS

ISSN 26832070

Número 04

julio-diciembre 2021
Artículo: “Enseñanza del Derecho en la Pandemia: La clase magistral interactiva en la virtualidad"

Cristina Cázares Sánchez

DOI: https://doi.org/10.25009/ej.v0i04.2569

colores e incluso elementos de transición ya que pueden causar desánimo o aburrimiento al alumnado (Maroto, 2008).

\section{La estructura de una clase magistral interactiva}

¿Qué temas son pertinentes de abordar en una clase magistral interactiva? Los temas del área de especialización o experiencia del Docente, en el ámbito virtual, este tipo de clases deben de plantearse como inicio de una unidad, un nuevo tema, no son un recurso cotidiano para las sesiones sincrónicas, debido al agotamiento que implica tanto para el docente como para el alumnado.

La clase magistral interactiva virtual o sincrónica tendrá como objetivo transmitir ese saber del docente en el tema de su dominio:

...las contribuciones del docente tienen como referente el conocimiento académico del tema, sin olvidar otras informaciones relacionadas con la enseñanza de la lección (formación y experiencia); en cuanto a los estudiantes, su referente son los conocimientos y experiencias previos de que disponen. El control para ajustar momento a momento la producción/comprensión de las contribuciones se ha de ejecutar en el plano de la preparación de lo que se transmite y en el comunicado recibido del interlocutor... (Tronchoni, Izquierdo, Anguera, 2018: 87)

¿Cómo crear el ambiente interactivo en una sesión sincrónica o presencial?

Para lograr el control de ajuste el docente utilizará recursos tecnológicos bajo la metodología de actividades donde el estudiante realice tareas como contestar preguntas breves o reflexione sobre pequeños casos prácticos o ejemplos, las respuestas se pueden obtener en tiempo real mediante aplicaciones tecnológicas gratuitas como kahoot o Answer Garden. También pueden enlazarse a las herramientas Moodle de las Ambientes Virtuales de Aprendizaje (EVA).

Para ello siguiendo la metodología de San Martín, Heurtebize, Veyrunes y Ría, 2021, respecto a una fase de trabajo individual y otra de trabajo colectiva para resolver el cuestionamiento planteado por el docente en la clase. En una sesión sincrónica virtual quedaría aplicado, de la siguiente manera:

A) El trabajo individual

Después de la fase expositiva anteriormente señalada con los elementos discursivos y argumentos vistos. Con una duración recomendada, aunque no exhaustiva de 15 minutos, se realiza la primera dinámica o actividad referida a los conceptos expuestos.

Para ello, puede utilizarse el planteamiento de un breve caso, con opciones de respuesta a través de Kahoot, que se contesta de forma individual o bien, una pregunta directa,

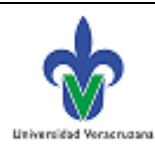

pp. $27-38$ 
ENFOQUES JURÍDICOS

REMSTA MULTIDISCIPLINAR DEL CEDEGS

ISSN 26832070

Número 04

julio-diciembre 2021
Artículo: “Enseñanza del Derecho en la Pandemia: La clase magistral interactiva en la virtualidad"

Cristina Cázares Sánchez

DOI: https://doi.org/10.25009/ej.v0i04.2569

contestada por Answergarden, de forma directa, personal y anónima que permitirá al docente observar la respuesta más frecuente en tiempo real y a través de la función de compartir pantalla, la podrá mostrar a la clase.

Dependiendo de la respuesta, puede dar una segunda fase expositiva el docente, si la respuesta más frecuente no fue la correcta.

B) El trabajo colaborativo

Una vez concluida la fase individual y la segunda exposición del docente, de ser el caso, se recomienda realizar una segunda actividad en equipo o en grupos, según la plataforma sincrónica que se utilice, que puede complejizarse para trabajar en la discusión de las respuestas de la anterior actividad o bien, en aumentar el objetivo o grado de profundidad de reflexión o aprendizaje (Ramsden, 2003).

Nuevamente pueden volver a utilizarse herramientas tecnológicas, solo que más complejas como un esquema en Google Drive o una breve presentación utilizando cualquier otra herramienta. También puede replantearse la pregunta usando Kahoot o AnswerGarden.

Dependiendo de las respuestas, el docente puede realizar una nueva fase expositiva del tema.

D) La evaluación y evidencias

Una de las grandes preocupaciones del docente, es la evaluación y los productos que servirán de evidencia para comprobar las actividades del docente, además de la evaluación formativa (García y Nicolás, coord., 2012), estas sirven para asignar una calificación.

Las evidencias de la interacción en clase quedan grabadas en la sesión sincrónica o bien puede establecerse una captura de pantalla, ya que las herramientas tecnológicas en el nivel básico gratuito por lo general no aplican para guardar la evidencia.

E) Fase de conclusión y plenaria

Por último, se debe realizar una actividad de cierre o conclusión en plenaria para retomar las dudas y diferencias entre las respuestas obtenidas en la fase de trabajo individual y en la fase de trabajo colaborativo.

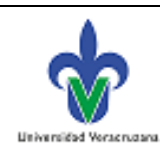

pp. 27-38 
ENFOQUES JURIDICOS

REVISTA MULTIDISCIPLINAR DEL CEDECS

ISSN 26832070

Número 04

julio-diciembre 2021
Artículo: “Enseñanza del Derecho en la Pandemia: La clase magistral interactiva en la virtualidad"

Cristina Cázares Sánchez

DOI: https://doi.org/10.25009/ej.v0i04.2569

\section{Conclusiones}

La clase magistral interactiva es un recurso funcional para la Educación en Línea derivada de la emergencia sanitaria, aun cuando podría clasificarse de tradicional este método de enseñanza, con la pausas, argumentos, lenguaje no verbal y estrategias de aprendizaje diseñadas por el docente, se puede fomentar la participación del estudiante para lograr un acercamiento cognitivo a los conceptos del Derecho, en particular, aquéllos que requieren de un grado de memorización por pertenecer a la teoría dogmática del Derecho.

La clase magistral permite conocer el enfoque del docente pero enriquecerlo con la participación de los alumnos, a través de la transmisión de emociones, sentimientos, experiencia y vivencias de los profesores que las imparten, por ello, esta estrategia puede, más allá de la transmisión del conocimiento de una clase magistral tradicional, trascender en un aprendizaje social para el alumnado como: recursos de oratoria, posturas y manejo de emociones a partir de la observación hacia los enseñantes.

Este tipo de estrategia se debe reservar a los temas que son áreas de especialización de los docentes, para darle un enfoque profundo de análisis y reflexión a los ejercicios de participación de los aprendientes, por ello, las clases magistrales interactivas son una más de las posibilidades en el abanico de estrategias docentes, no deben convertirse en la forma habitual en las clases en línea dentro del contexto mundial actual. 
ENFOQUES JURÍDICOS

REMSTA MULTIDISCIPLINAR DEL CEDECS

ISSN 26832070

Número 04

julio-diciembre 2021
Artículo: “Enseñanza del Derecho en la Pandemia: La clase magistral interactiva en la virtualidad"

Cristina Cázares Sánchez

DOI: https://doi.org/10.25009/ej.v0i04.2569

\section{Fuentes de consulta}

Amilcar, María Julia, María Martha Laguyás y Leonardo P. Palacios (2011) Posibilidades Metodológicas en la Enseñanza del Derecho, ANALES № 41 Facultad de Cs. Jurídicas y Sociales. U.n.I.p. pp 317-328

Badia, Antoni (2005) El uso educativo de las aulas virtuales emergente en la educación superior, Revista de la Universidad y Sociedad del Conocimiento, vol 2, n2.

Charaja Cutipa, Francisco (2014) Vigencia de la clase magistral en la universidad del siglo XXI, Apuntes Universitarios, Revista de Investigación, Universidad Peruana, vol. IV, núm 1, enero-junio, pp. 57-66.

Garcia y García, Noemí y Nicolás Mora, Rosa María, Coord. (2012) El enfoque formativo de la evaluación, Serie: Herramientas para la evaluación en educación básica, SEP. pp. 67.

Elgueta Rosas, María Francisca y Palma González, Eric Eduardo (2014) Revista Chilena de Derecho, vol 41, N3, pp. 907-924.

Hodelín Hodelín, Yasnay, De los Reyes García, Zaida, Hurtado Cumbá, Gleidis, Batista Salmon, Milton (2016) Riesgos sobre tiempo prolongado a un ordenador, Revista de Información Científica (Internet), 175-190. Recuperado de http://www.revinfcientifica.sld.cu/index.php/ric/article/view/149/1447

Maroto Marín, Orlando (2008) El uso de las presentaciones digitales en la educación superior: una reflexión sobre la práctica, Revista Electrónica "Actualidades Investigativas en Educación", vol 8, núm 2, mayo-agosto, pp. 1-21.

Morell Moll, Teresa (2014) La interacción en la clase magistral, Universidad de Alicante, España.

Ramello, Ames. P.P. (2019) El uso de materiales audiovisuales y recursos digitales en la docencia universitaria: una experiencia de innovación a nivel de posgrado en Perú, Revista de docencia universitaria, vol 17(1) enero-junio, pp. 167-182.

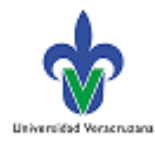

pp. 27-38 
ENFOQUES JURÍDICOS

REVISTA MULTIDISCIPLINAR DEL CEDECS

ISSN 26832070

Número 04

julio-diciembre 2021
Artículo: “Enseñanza del Derecho en la Pandemia: La clase magistral interactiva en la virtualidad"

Cristina Cázares Sánchez

DOI: https://doi.org/10.25009/ej.v0i04.2569

Ramos Navas Parejo M., Rodríguez García, M.A., Gómez García, G. (2019). La gamificación en el aula universitaria. Una metodología activa e inclusiva. Percepciones por parte del alumnado universitario, Investigación e innovación en la Enseñanza Superior: Nuevos contextos, nuevas ideas / coord. por Rosabel Roig Vila, 2019, ISBN 978-8417667-23-8, págs. 669-678.

Ramsden, Paul. (2003). Learning to Teach in Higher Education, 2nd Edition, RoutledgeFalmer. pp. 288.

Renés Arellano, Paula (2018). Planteamientos de los Estilos de Enseñanza desde un enfoque cognitivo constructivista. Tendencias pedagógicas, ISSN 1133-2654, № 31, 2018, págs. 47-68.

San Martín, Julia, Heurtebize Sylvia, Veyrunes Philippe, Ría Lucía (2021) Convergences et divergences entre l'activité des enseignants et celle des étudiants lors de cours magistraux interactifs: une étude exploratoire, en prensa.

Sánchez-Carracedo, Fermín y Barba Vargas, Alejandra (2019) Como impartir una clase magistral según la neurociencia, Actas de la Jenui, vol 4, pp. 87-94.

Shablico, Sandra (2012) La comunicación no verbal en el aula, un análisis en la enseñanza disciplinar, Cuadernos de Investigación Educativa, vol 3, núm 12, pp. 99-121.

Tarabay, Fanny. (2007). Estrategias argumentativas en el discurso docente de la clase magistral universitaria, Gestión y Gerencia, Revista Científica, Decanato de Administración y Contaduría, Universidad Centroccidental “Lisandro Alvarado", pp. 27-37.

Tronchoni, Héctor, Izquierdo, Conrad, Anguera, María Teresa. (2018). Interacción participativa en las clases magistrales: Fundamentación y construcción de un instrumento de observación, Publicaciones, 48(1), pp. 81-108.

Unidad de Innovación Educativa (2020) Guía pedagógica para el desarrollo de clases en entornos virtuales, Universidad de Santiago de Chile. 